\title{
Isolated Cervical Cord Involvement in Primary Central Nervous System Vasculitis
}

CHRONG-REEN WANG, MD, PhD, Department of Internal Medicine; SHU-LING PENG, MD, Department of Pathology; HAN-WEI HUANG, MD, Department of Neurology, National Cheng Kung University Hospital, Tainan, Taiwan. Address correspondence to Dr. C.R. Wang, Section of Rheumatology, Department of Internal Medicine, National Cheng Kung University Hospital, No. 138 Sheng-Li Road, Tainan 70403, Taiwan. E-mail: wangcr@mail.ncku.edu.tw. The Institutional Review Board of the hospital approved this study (B-ER-105-108). J Rheumatol 2018;45:962-3; doi:10.3899/jrheum.171212

Spinal cord involvement has been observed rarely in primary central nervous system vasculitis (PCNSV), and histopathologically proven isolated cord angiitis usually involves the thoracic level or terminal conus ${ }^{1,2}$. We report a very rare case of isolated cervical cord involvement in PCNSV. The diagnosis was first made by spinal magnetic resonance imaging (MRI) to survey the possible etiologies of cervical myelopathy, and finally confirmed by histopathological analysis.

A 57-year-old Han Chinese male was transferred to the Internal Medicine Department of National Cheng Kung University Hospital in Taiwan in September 2012 with an 8-month history of quadriparesis. He had a diagnosis of cervical myelopathy because of progressive 4-limb weakness and paresthesia, as well as neurogenic bladder. Autoantibody profiles were negative. Spinal MRI showed focal intramedullary enhancing lesions at C3-6 levels with cord edematous change (Figure 1A), leading to a possible cause of myelitis, demyelination, or tumor. Further, brain MRI examination showed no abnormality. Nevertheless, histopathological analysis revealed perivascular granulomatous lesion and lymphocyte infiltration composed of clustered epithelioid histiocytes and aggregated lymphocytes, consistent with granulomatous angiitis (Figure 1B). There were no vascular thrombosis, stained pathogens, and demyelinating lesions in the cord sections. Indeed, PCNSV imaging may show enhancing lesions, such as in our case ${ }^{3}$. High-dose corticosteroids $(1 \mathrm{mg} / \mathrm{kg} /$ day prednisolone $)$ and monthly pulse cyclophosphamide (CYC) therapy $\left(0.75 \mathrm{~g} / \mathrm{m}^{2}\right.$ for 6 mos) were prescribed under the diagnosis of PCNSV ${ }^{4}$, followed by maintenance usage of oral CYC $(1 \mathrm{mg} / \mathrm{kg} / \mathrm{day})$. Despite the reported recovery of neurological deficit by the corticosteroid/immunosuppressant therapy ${ }^{5}$, our patient had stationary quadriparesis without other organ involvement during a 5-year followup period.

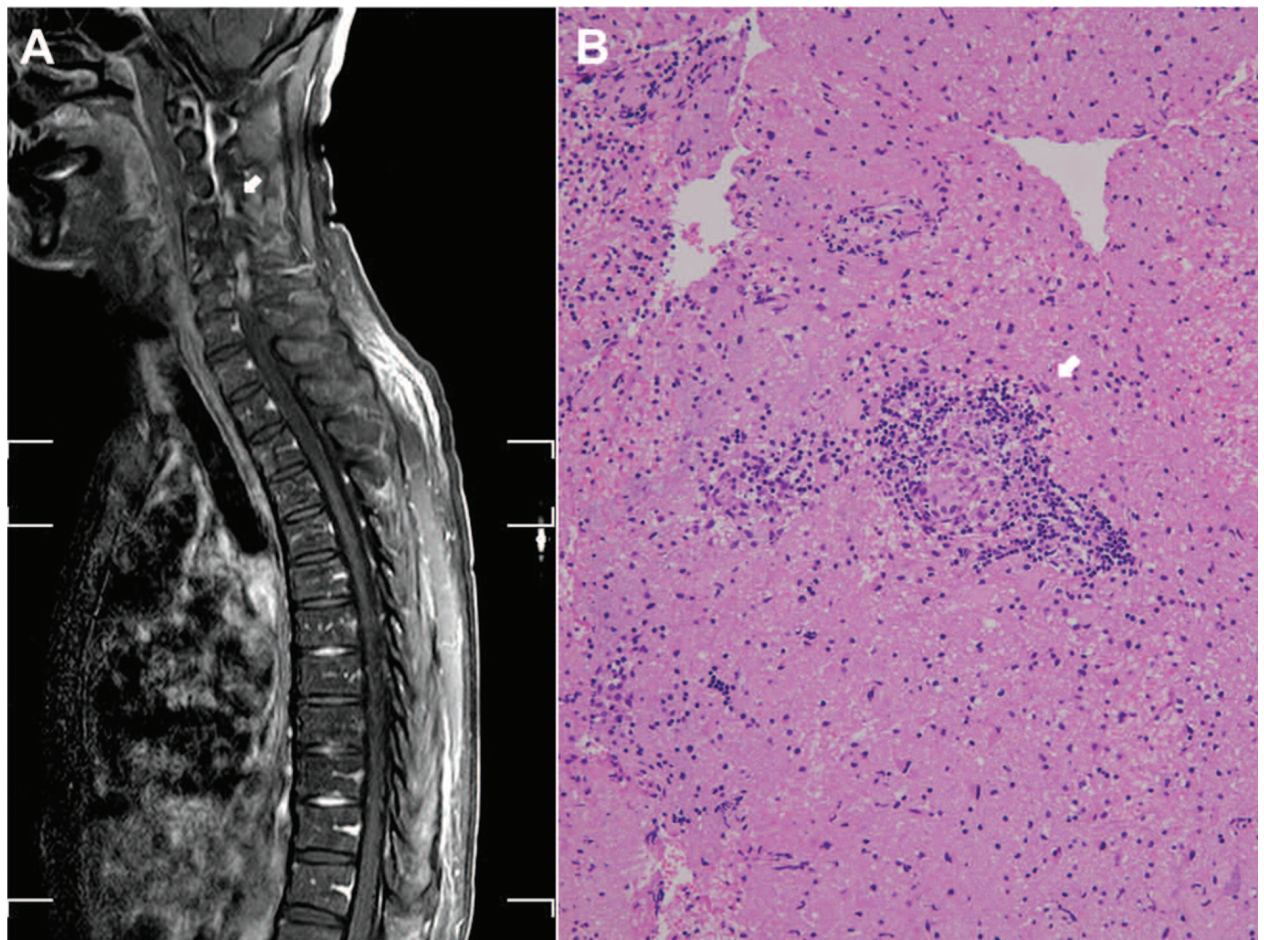

Figure 1. Spinal MRI and histopathological analyses. A. Sagittal T1-weighted, fat-suppressed, gadolinium-enhanced spinal MRI showing intramedullary enhancing lesion at C3-6 levels (white arrow). B. Histopathological findings of spinal cord tissues revealing gliosis change and granulomatous angiitis with a small artery (central area, white arrow) containing perivascular granulomatous lesion and lymphocyte infiltration consisting of clustered epithelioid histiocytes and aggregated lymphocytes (H\&E staining, 200×). MRI: magnetic resonance imaging. 


\section{REFERENCES}

1. Salvarani C, Brown RD Jr, Calamia KT, Christianson TJ, Huston J 3rd, Meschia JF, et al. Primary CNS vasculitis with spinal cord involvement. Neurology 2008;70:2394-400.

2. Néel A, Pagnoux C. Primary angiitis of the central nervous system. Clin Exp Rheumatol 2009;27(1 Suppl 52):S95-107.
3. Abdel Razek AA, Alvarez H, Bagg S, Refaat S, Castillo M. Imaging spectrum of CNS vasculitis. Radiographics 2014;34:873-94.

4. Salvarani C, Brown RD Jr, Hunder GG. Adult primary central nervous system vasculitis. Lancet 2012;380:767-77.

5. Goertz C, Wegner C, Brück W, Berlit P. Primary angiitis of the CNS with pure spinal cord involvement: a case report. J Neurol 2010;257:1762-4 